\title{
Human Papillomavirus Genotypes in the High Grade Preinvasive Cervical Lesions in Yaounde (Cameroon)
}

\author{
Zacharie Sando $^{1, *}$, Tomas McKee ${ }^{2}$, Jean Claude Pache ${ }^{2}$, Herman Kemajou ${ }^{1}$, Olive Folem ${ }^{1}$, Laura Rubbia - \\ Brandt $^{2}$, Anderson Sama Doh ${ }^{1}$ \\ ${ }^{1}$ Department of Pathology, Gynaeco-obstetrics and Pediatric Hospital, Yaoundé, Cameroon \\ ${ }^{2}$ Department of Clinical Pathology, Geneva University Hopsital, Geneva, Switzerland \\ *Corresponding author: sandozac@yahoo.fr
}

Received December 29, 2013; Revised August 19, 2013; Accepted August 21, 2013

\begin{abstract}
The objectives of this study were to detect and determine the different genotypes of Human Papillomavirus (HPV) found in high-grade squamous preinvasive lesions of the uterine cervix of Cameroonian women. HPV genotyping was conducted on 37 endocervical secretions of women with high grade squamous intraepithelial lesions. HPV was found in 31 of the 37 women (83.8\%); 9 different HPV genotypes were identified. The genotypes with high oncogenic potential were found in decreasing order of frequency as follows: $16,18,45,33$, 35 and 68. The frequency of strains found per patient ranged from 1 to 3 . Genotypes 16 and 18 were single in 17 out of 31 patients. They were associated with other HPV genotypes in 4 out of 31 patients. In this study genotypes 16 and 18 are the most frequent genotypes encountered in high grade squamous preinvasive cervical lesions in Yaounde. This finding, if confirmed on a larger sample, portrays the potential effectiveness of HPV vaccines in the Cameroonian population as a preventive measure against cervical cancer. However, the position of genotype 45 is not negligible and should be confirmed on a large sample.
\end{abstract}

Keywords: human papillomavirus, high grade preinvasive lesion, cervix

Cite This Article: Zacharie Sando, Tomas McKee, Jean Claude Pache, Herman Kemajou, Olive Folem, Laura Rubbia - Brandt, and Anderson Sama Doh, "Human Papillomavirus Genotypes in the High Grade Preinvasive Cervical Lesions in Yaounde (Cameroon)." American Journal of Cancer Prevention 1, no. 3 (2013): 20-23. doi: 10.12691/ajcp-1-3-1.

\section{Introduction}

Cancer of the uterine cervix is today one of the biggest concerns of the medical community. It is the second most common gynecological cancer in women worldwide [1]. Each year, approximately 400,000 to 500,000 new cases of cervical cancer are diagnosed and are the cause of about 200,000 to 300,000 deaths [1]. About $80 \%$ of these cancers occur in developing countries, particularly in subSaharan Africa, in contrast to developed countries where frequency and mortality from this cancer is on the decrease [1].

In Yaounde Cameroon, it is the second most common cancer in women with a prevalence of $21.4 \%$ after breast cancer which accounts for $30.4 \%$ [2]. Invasive cancer is generally preceded by 10 to 15 years of precancerous lesions that are asymptomatic. These lesions represent the intermediate stage between normal cervical epithelium and invasive cervical cancer. Amongst them there are the low-grade lesions corresponding to cervical intraepithelial neoplasia of the first degree (CIN1) and high-grade lesions corresponding to cervical intraepithelial neoplasia of the second and third degrees (CIN2, CIN3). Between one to three years, the CIN1, CIN2 and CIN3 regress spontaneously in 57\%, $43 \%$ and $32 \%$ of women respectively. Conversely, they progress to cancer in $1 \%$, $1.5 \%$ and $12 \%$ for CIN1,CIN2 and CIN3 respectively [3]. There are many co-factors involved, but the Human Papillomavirus (HPV) is the etiological factor and is present in more than $99.7 \%$ of cervical cancers [4]. The genotypes of the virus can be classified according to their oncogenic potential (low or high). Persistent infection with highly oncogenic HPV is a necessity for progression to invasive cancer. There is an unequal distribution of HPV genotypes according to geographical area [5,6,7]. For example, there is a high prevalence of HPV type 16 in the Western world in contrast to Africa [1,5,6,7]. In Latin America and the Caribbean, multiples HPV are found in of high grade lesions with 46, $5 \%$ harboring HPV 16 and 8,9 \% HPV 18 [8]. Multiple HPV genotypes have been also found in high grade lesions and cancer in America with HPV 16 the most frequent at all disease categories [9]. HPV vaccines currently proposed target only two highly oncogenic genotypes: 16, 18 and two genotypes of low oncogenicity: 6 and 11. Several studies of HPV genotype profile have been carried out in the world but very few in Cameroon. Most of these studies targeted the genotypes 16 and 18, and were done on the general female population, not including the fact that the attribution of HPV vaccine genotypes varies from a category of cervical disease to another [9]. In the absence of epidemiological data on the distribution of HPV genotypes in Cameroon, 
namely in high-grade squamous intraepithelial lesions that are most at risk of becoming cancerous, we carried out a descriptive study of HPV genotypes in female patients with high-grade cervical lesions.

\section{Materials and Methods}

\subsection{Site, Study Period and Setting}

Recruitment of cases and data collection was done in the Department of pathology of the Yaoundé GynaecoObstetrics and Pediatric Hospital (YGOPH) in Cameroon, from $1^{\text {st }}$ January to $31^{\text {st }}$ December 2009, among patients referred from the Gynecology Department for screening of precancerous lesions of the uterine cervix.

\subsection{Criteria for Inclusion and Exclusion}

Patients included in the study were aged at least 25 years from whom we obtained informed consent and whose cervical smears showed high grade precancerous lesions diagnosed on conventional smears (in Cameroon) or thin Prep (in Geneva) with histopathological confirmation from biopsy. Specimens from women undergoing surgery, radiotherapy or chemotherapy for gynecological cancer were excluded.

\subsection{Samples Taken and Laboratory Methods}

\subsubsection{Collection of Cervical Smears}

Patients received in the examination room were positioned in the gynecologic position on the examination table. After introduction of a non-lubricated, clean and single-use speculum (MediWare ${ }^{\circledR}$ ), the endo-cervical secretions were collected using a cytobrush (Cervex-Brush ( R Rovers Medical Devices BV Lekstraat 105347 KV Oss, the Netherlands). After spreading the conventional pap smear on a glass slide, the head of the cytobrush with the specimen obtained by brushing the cervix was separated from its handle and then put into a vial containing a biological fixative (Cytix ${ }^{\circledR}$ ) and labeled for identification of sampling. All the specimens were shipped at $20^{\circ} \mathrm{C}$ using dry ice, according to international standards within a period of three weeks.

\subsubsection{Cytopathological Examination}

Cytopathological examination of the samples from the patients was done after Papanicolaou stain procedure of conventional pap smears in the Pathology Department of the YGOPH, Yaounde Cameroon by a certified cytotechnician and certified cytopathologist. Thin Prep slides as well as the slides from biopsies were reviewed in the clinical pathology department of the University of Geneva, Switzerland by certified cytopathologists. The cytological abnormalities were classified according to Bethesda classification system (2001).

\subsubsection{Detection of HPV}

High grade cervical lesions diagnosed either in Thin Prep in Geneva or conventional Pap smear in Cameroon with histopathological confirmation from biopsies were enrolled in HPV detection. Detection and genotyping of HPV took place at the Department of Pathology,
University Hospital of Geneva, under the supervision of Dr Tomas Mckee. An aliquot of the cervical sample was processed as described previously by Kuypers et al [10]. Briefly, the samples were precipitated and centrifuged, and the pellet dried and resuspended. HPV detection and typing was carried out using a polymerase chain reaction(PCR-), based reverse-line strip test for HPV types 6, 11, $16,18,26,31,33,35,39,40,42,45,51-59,66,68,73,82$, 83 , and 84 as previously described [11]. B-globin was used as an internal control [11].

\subsubsection{Ethical Considerations}

The study was approved by both review board of Cameroon National Ethics Committee and the Geneva University Hospitals Ethics committee, Switzerland.

\section{Results}

Thirty nine women were enrolled in the study. Thirty seven specimens cytologically diagnosed as high grade squamous intraepithelial lesions (HGSIL) both in thin Prep and conventional smear, with histological confirmation of CIN2 or CIN3 were included in our study. There was a discrepancy in one case ( ASCUS-H) with histopathological diagnosis of CIN1-2 and was excluded. Out of the 37 specimens retained, the HPV test was positive in 31 of the 37 women (83.8\%). A total of 39 HPV isolates were identified amongst which we noted nine different HPV genotypes. The majority of these isolates $(89.7 \%)$ ) were high risk (HR-HPV) including types 16 and 18 which accounted for $58.9 \%$ of the isolates and occupied respectively the first and second rows (Figure 1). Of the nine types of HPV identified, six genotypes were identified as HR-HPV and in descending order as follows: HPV16 (30.7\%), HPV18 (28.2\%), HPV45 (15.3\%), HPV33 (7.6\%), HPV35 (5.1\%), and HPV 68 (2.5\%); 3 genotypes were low risk (LR HPV) and in decreasing frequency as follows: HPV 11 (5.1\%), HPV 53 (2.5\%), and HPV 74 (2.5\%).

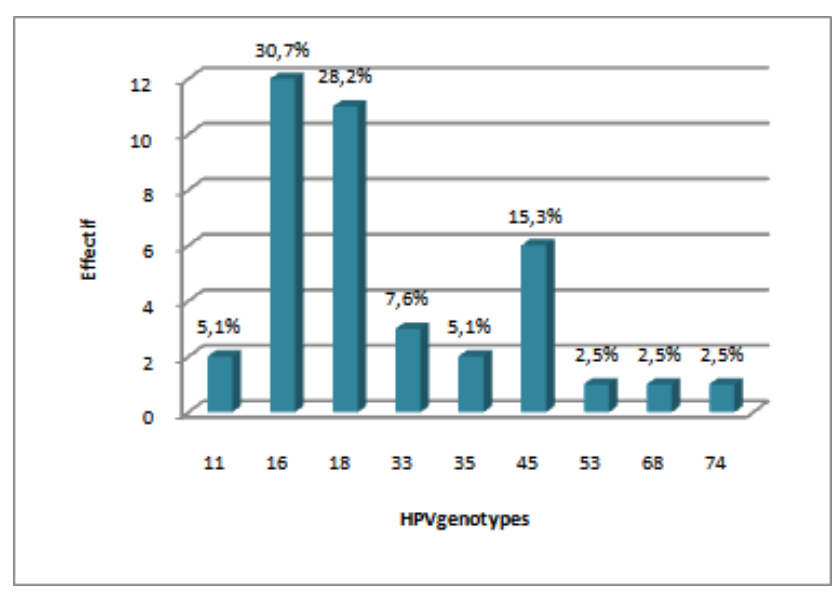

Figure 1. Overview of the different genotypes encountered

Of the 31 women identified as carriers of highoncogenic HPV, 28/31 (90.3\%) were carriers of HR HPV and $3 / 31(9,7 \%)$ were infected with LR HPV.

Genotypes 16 and 18 that are used in the current HPV vaccines were single in 17out of 31 (54.8\%) patients and were associated to other HPV types in 4out of 31 (12.9\%) patients. The frequency of the HPV types found by 
women ranged from 1 to 3 . Of the 31women with positive with HR- HPV test twenty six (83.9\%) had one genotype, two (6.4\%) 2 genotypes, and three (9.6\%) 3 genotypes (Table 1).

Table 1. Distribution of high-grade lesions according to the HPV genotype

\begin{tabular}{ccc}
\hline HPV & \multicolumn{2}{c}{ High grade lesion (H-SIL) } \\
\cline { 2 - 3 } One genotype & Frequency & Percentage \\
11 & 2 & 6,5 \\
16 & 9 & 29,0 \\
18 & 8 & 25,8 \\
33 & 2 & 6,5 \\
35 & 1 & 3,2 \\
45 & 3 & 9,7 \\
68 & 1 & 3,2 \\
Two genotypes & & \\
$16-45$ & 1 & 3,2 \\
$18-45$ & 1 & 3,2 \\
Three genotypes & & 3,2 \\
$16-18-45$ & 1 & 3,2 \\
$16-18-33$ & 1 & 3,2 \\
$53-35-74$ & 1 & \\
\hline
\end{tabular}

\section{Discussion}

In Cameroon some studies have been done on the awareness, knowledge, and belief on HPV, cancer and HPV vaccines, but no study on HPV detection in the high grade cervical lesions $[12,13,14,15]$. In our study, viral DNA was found in $83.8 \%$ cervical samples of the women. This result is similar to that found by Hyo Sung et al in Korea (89.4\%) [16]. On the contrary, our finding is higher than that of Didelot-Rousseau et al in 2006 in Burkina Faso, Stevens $\mathrm{M}$ et al in Australia and Lindemann et al in Spain, who respectively had $66.1 \%, 68.35 \%$ and $65.4 \%$ in their studies $[5,17,18]$.

This difference in prevalence in patients who had highgrade lesions could be explained by several factors, namely the viral genotyping procedure, and the detection technics used in these studies which require that the viral DNA be qualitatively and quantitatively satisfactory. Some technics requires a minimum of 10 to 100 copies of DNA for the sample to be positive. It is also possible that in some cases, the DNA could not be detected because of its poor quality. The sensitivity could attain $100 \%$ when the DNA is qualitatively adequate. Finally, there are cases where some factors as smoking and HIV infection are very important co-factors for progression to high-grade lesions than HPV alone [5,19].

We identified 9 types of HPV genotypes in 39 isolates of HPV. This result is much lower than that from Burkina Faso, where Didelot Rousseau et al identified 35 HPV genotypes [5]. This could be explained by our small sample size.

Other studies have shown that the distribution of HPV types was highly variable from one country to another $[5,6,7,8,9]$. The different HPV genotypes most frequently encountered in our study appeared to be similar to those found in high-grade precancerous lesions in the literature. Genotypes 16, 18, 45, 33, 35, 31 and 58 are present in most high-grade squamous intraepithelial lesions and $89 \%$ of cervical cancer [8,20,21].

Our results indicate that genotypes 16 and 18 are the most frequent genotypes (58.9\%), and this is similar to findings from other studies Lindemann et al at the Spanish cohort found that the 16 and 18 HPV genotypes were responsible for $65.4 \%$ of high-grade lesions [18]. Ciapponi et al found from a systematic review and metaanalysis in Latin America and the Caribbean 46,5 \% of $\mathrm{H}-$ SIL with HPV16 and 8,9 \% with HPV18 [8]. Similarly in Burkina Faso, these genotypes were incriminated in 64\% of cases of high-grade lesions, although they represented a small proportion in the general population [5]. A variant of phylogenetic HPV 16 or 18 was found in $93 \%$ of women with high-grade lesions [5]. On the contrary Chen et al in Taiwan in 2006 reported a low frequency of these two genotypes. He found then in only $33.4 \%$ of the genotypes encountered. The genotypes found in descending order were: 16 (31.7\%), 58 (20\%), 52 (19.7\%), 33 (12.8\%), 51 (3.4\%), 31 ( 2.4\%), 59 (2.1\%), 53 (1.7\%), 18 (1.7\%), 39 (1\%), 45 (1\%), 66 (0.3\%) [15]. In these studies genotype 16 was also the most prevalent. This was followed by genotypes 31 and 58 in Spain [21], 52 and 58 in Taiwan [22]. In our study, genotype 18 was the second most prevalent genotype with a frequency much higher than that found in other localities. Lindermann et al, in Spain found a low prevalence of genotype 18 (5.8\%) in the eight position in all the genotypes encountered [18]. Similarly, in Taiwan this genotype occupied the eight position, and accounted for $1.7 \%$ of all the genotypes encountered [22]. An under-estimation of genotype 18 in the HGSIL is noted in some studies [9,20].

Desruissseau et al reported a study done in two cities in Cameroon which revealed high frequencies of genotypes 45 and 58 in a general population. In this study, the frequency of genotypes 16 and 18 appeared to be lower [23].

Our study on the contrary revealed a higher frequency of these genotypes probably because it was carried out on women with high-grade lesions. Of the 39 isolates identified, 89.2\% were HPV with high oncogenic potential and were present in $90.3 \%$ of the women. These results are consistent with those of Hyo Sung et al, who found in his cohort 55 out of 59 (83, 3\%) women with HPV genotypes of high oncogenic potentials [8].

The proportion of patients in our study who had genotypes found in vaccines corroborates those of Burkina Faso where Didelot-Rousseau et al found genotypes 16 and 18 in 64\% of women with high-grade lesions [5]. Yet these two genotypes are generally less frequent in the population- based study that included preinvasive lesions of different grades [5,9].

\section{Conclusion}

Our finding reveals a significant potential effectiveness of available anti HPV vaccines targeting HPV 16 and 18 in the Cameroonian population to reduce cervical cancer. But further studies on a larger sample are necessary to confirm this observation. However, the position of HPV genotype 45 is not negligible and should be looked for specifically in further studies with larger sample sizes of high grade cervical lesions.

\section{References}

[1] Forouzanfar MH, Foreman KJ, Delossantos AM, Lozano R, Lopez $\mathrm{AD}$, Murray CJL et al. Breast and cervical cancer in 187 countries 
between 1980 and 2010: a systematic analysis. The lancet, 378 (9801):1461-1484, Oct. 2011.

[2] Enow-Orock GE, Essame-Oyono JL, Doh A . Cancer incidence in Yaoundé 2004-2008: Yaoundé Cancer Registry Technical Report.Yaoundé: National Cancer Control Program, Yaounde Cameroon, 2009.

[3] Sellors JW, Sankaranarayanan R. Colposcopie et traitement des néoplasies cervicales intraépithéliales: Manuel à l'usage des débutants. Centre international de recherche sur le cancer, Lyon France, 2004.

[4] Walboomers JMM, Jacobs MV, Manos MM, Bosch FX, Kummer $\mathrm{JA}$, Shah KV et al. Humann papilloma virus is a necessary cause of invasive cervical cancer worldwide. J Pathol, 189 (1):12-19, Sept. 1999.

[5] Didelot-Rousseau M-N, Nagot N, Costes-Martineau V, Ouedraogo A, Konate I, Weiss HA et al. Human papillomavirus genotypes distribution and cervical intraepithelial lesions among high-risk women with and without HIV-1 infection in Burkina Faso. $\mathrm{Br} \mathrm{J}$ Cancer, 95 (3): 355-362, Aug. 2006.

[6] Ali-Risasi C, Praet M, Van Renterghem L, Zinga-Ilunga B, Sengeyi D, Lokomba V et al. Human Papilloma virus genotypic Profile in Kinshasa, Democratic Republic of Congo : implications for vaccination. Med Trop, 68(6) : 617-20, Dec. 2008.

[7] Salih MM, Safi ME, Hart K, Tobi K, Adam I. Genotypes of human papilloma virus in Sudanese women with cervical pathology, Infect Agent Cancer, 5: 26, Dec. 2010.

[8] Ciapponi A, Bardach A, Glujovsky D, Gibbons L, Picconi MA. Type-specific HPV prevalence in cervical cancer and high-grade lesions in Latin America and the Caribbean: systematic review and meta-analysis. PLoS One 6 (10):e25493. Epub 2011 Oct. 4.

[9] Wentzensen N, Schiffman M, Dunn T, Zuna RE, Gold MA, Allen RA et al. Multiple human papillomavirus genotype infections in cervical cancer progression in the study to understand cervical cancer early endpoints and determinants. Int $J$ Cancer, 125 (9):2151-8, Nov. 2009

[10] Kuypers JM, C. W. Critchlow CW, Gravitt PE, Vernon DA, Sayer JB, Manos MM et al., Comparison of dot filter hybridization, southern transfer hybridization, and polymerase chain reaction amplification for diagnosis of anal human papillomavirus infection. J Clin Microbiol, 31(4):1003-6, Apr. 1993.

[11] Gravitt PE, Peyton CL, Apple RJ, Wheeler CM. Genotyping of 27 human papillomavirus types by using L1 consensus PCR products by a single-hybridization, reverse line blot detection method, $J$ Clin Microbiol, 36 (10):3020-7, Oct. 1998.

[12] Wamai RG, Ayissi CA, Oduwo GO, Perlman S, Welty E, Manga $S$ et al. Assessing the effectiveness of a community-based sensitization strategy in creating awareness about HPV, cervical cancer and HPV vaccine among parents in North West Cameroon. J Community Health, 37(5):917-26, Oct. 2012.

[13] Ayissi CA, Wamai RG, Oduwo GO, Perlman S, Welty E, Welty T et al. acceptability and uptake of human papilloma virus vaccine among Cameroonian school-attending female adolescents. $J$ Community Health, 37(6):1127-35, Dec. 2012.

[14] McCarey C, Pirek D, Tebeu PM, Boulvain M, Doh AS, Petignat P. Awareness of HPV and cervical cancer prevention among Cameroonian healthcare workers. BMC Womens Health , 11:45, Oct. 2011.

[15] Berner A, Hassel SB, Tebeu PM, Untiet S, Kengne-Fosso G, Navarria I et al. Human Papillomavirus Self-Sampling in Cameroon: Women's Uncertainties Over the Reliability of the Method Are Barriers to Acceptance. J Low Genit Tract Dis, 17(3): 235-41, Jul. 2013.

[16] Hwang HS, Park M, Lee SY, Kwon KH, Pang MG. Distribution and Prevalence of Human Papillomavirus Genotypes in Routine Pap Smear of 2470 Korean Women Determined by DNA chip. Cancer Epidemiol Biomarkers Prev, 13 (12):2153-56, Dec. 2004.

[17] Stevens MP, Tabrizi SN, Quinn M , Garland SM. Human papillomavirus genotype prevalence in cervical biopsies from women diagnosed with cervical intraepithelial neoplasia or cervical cancer in Melbourne, Australia. Int J Gynecol Cancer, 16 (3): 1017-1024, May-Jun. 2006

[18] Lindemann MLM, Sanchez JMC, Chacon JA, Itziar S, Diaz E, Rubio MD et al. Prevalence and distribution of high-risk genotype of HPV in women with severe cervical lesion in Madrid, Spain: importance of detecting genotype 16 and other high-risk genotypes. Adv Prev Med 2011; 2011:269468. doi: 10.4061/2011/2694 Epub 2010 Sep. 27.

[19] Winkelstein WJ. Smoking and cervical cancer-current status: a review. Am J Epidemiol, 131(6): 945-957, Jun. 1990.

[20] Clifford GM, Smith JS, Plummer M, Muňoz N. \& Franceschi S. Human papilloma virus types in invasive cervical cancer worldwide: a meta analysis. Br J Cancer, 88 (1):63-73, Jan. 2003.

[21] Muňoz N, Bosh F X, Casttellague X, de Sanjose S, Hammouda D, Shah K V \& al. Against which human papilloma virus types shall we vaccinate and screen? The international perspective. Int $J$ Cancer; 111(2):278-285, Aug. 2004.

[22] Chen CA, Liuy HH, Chou CY, Ho CM, Twu NF, Kan YY et al. The distribution and differential risks of human papilloma virus genotypes in cervical pre invasive lesions: a Taiwan Cooperative Oncologic Group Study. Int J Gynecol Cancer, 16 (5):1801-1808, Sept-Oct. 2006.

[23] Desruisseau AJ, Schmidt-Drimminger D, Welty E. Epidemiology of HPV in HIV-Positive and HIV-Negative Fertile Women in Cameroon, West Africa. Inf Dis Obstet Gynecol vol 2009 (2009). 